Gastroenterol 2006; 101: 812-22.

2 Munoz P, Bouza E, Cuenca-Estrella M, et al. Saccharomyces cerevisiae fungemia: an emerging infectious disease. Clin Infect Dis 2005; 40: 162534.

3 Enache-Angoulvant A, Hennequin C. Invasive Saccharomyces infection: a comprehensive review. Clin Infect Dis 2005; 41: 1559-68.

4 Lherm T, Monet C, Nougière B, et al. Seven cases of fungemia with Saccharomyces boulardii in critically ill patients. Intensive Care Med 2002; 28: 797-801.

5 Johnston BC, Supina AL, Vohra S. Probiotics for pediatric antibiotic-associated diarrhea: a meta-analysis of randomized placebo-controlled trials. CMAJ 2006; 175: 377-83.

\section{Application of normal saline to ultrasonography as an alternative to gel for internal jugular venous cannulation}

To the Editor:

Percutaneous central venous catheterization can be facilitated with real-time observation of vessels using 2-D ultrasonography. Various methods have been described when using this technique, ${ }^{1-4}$ each of which incorporates gel application to the referred skin area in order to ensure adequate delivery of the echo beam to the deep tissues. ${ }^{1}$ While the use of gel is common, its safety for central venous cannulation has not been clearly established. Furthermore, difficulties may be associated with catheter handling as gel solutions may make the catheter slip in the operator's hands, and there is potential for an increased infection rate. ${ }^{5}$ Saline may be an effective, less expensive alternative to gel.

Recently, for a patient undergoing central venous cannulation with ultrasonography, we compared images using sterile normal saline and gel on the referred skin surface. Ultrasonography of the right internal jugular vein (IJV) was performed using a GE Book XP (General Electrics Ltd, USA) equipped with an 8$\mathrm{MHz}$ probe, from which we obtained three different views. We first covered the probe tightly with a sterilized silicon glove containing gel. After covering the skin area with gel, we obtained a view that could distinguish between the carotid artery and the IJV (Figure, panel A). Next, the skin was thoroughly cleaned and the probe was re-applied without gel on the skin area. We could not obtain a clear image that would be suitable for IJV catheterization (Figure, panel B). Finally, we applied sterile normal saline over the skin, which

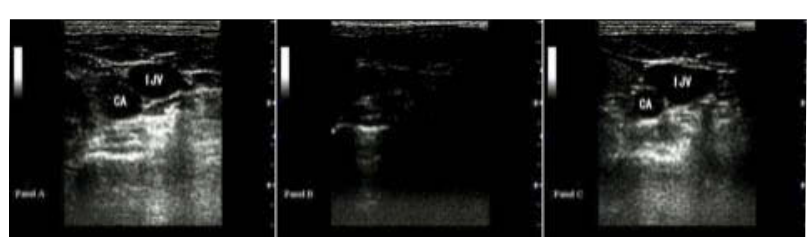

FIGURE Panel A) The view obtained by using gel on the skin surface (conventional method), where the CA and IJV are easily distinguished. The probe was lubricated with gel and then wrapped in a sterile glove. Panel B) View obtained without gel or saline. Panel C) View obtained with sterile normal saline applied to the skin surface. The CA and IJV are readily visualized. The gel covered only the surface of the probe within a sterile glove (it did not directly touch the skin surface). $\mathrm{CA}=$ carotid artery; $\mathrm{IJV}=$ internal jugular vein).

generated an ultrasonographic image (Figure, panel C) very similar to that obtained with the gel.

These observations suggest that a more detailed examination of normal saline as a substitute for skin application, compared to gel solutions to facilitate ultrasound-guided techniques for IJV cannulation, is warranted.

Hiroshi Dohgomori MD

Masato Shiba MD

Kazufumi Okamoto MD

Shinshu University Hospital, Asahi, Matsumoto, Japan

E-mail: hd5621hd@yahoo.co.jp

Accepted for publication December 19, 2006.

\section{References}

1 Chapman GA, Johnson D, Bodenham AR. Visualisation of needle position using ultrasonography. Anaesthesia 2006; 61: 148-58.

2 Milling T, Holden C, Melniker L, Briggs WM, Birkhahn $R$, Gaeta T. Randomized controlled trial of singleoperator vs. two-operator ultrasound guidance for internal jugular central venous cannulation. Acad Emerg Med 2006; 13: 245-7.

3 DiCarlo JV, Auerbach SR, Alexander SR. Clinical review: alternative vascular access techniques for continuous hemofiltration. Crit Care 2006; 10: 230.

$4 \mathrm{McGee} D C$, Gould MK. Complications of central venous catheterizations. N Engl J Med 2003; 348: 1123-33.

5 Ohara T, Itoh $\Upsilon$, Itoh $K$. Ultrasound instruments as possible vectors of staphylococcal infection. J Hosp Infect 1998; 40: 73-7. 